\title{
Simultaneous dual wavelength eye-tracked ultrahigh resolution retinal and choroidal optical coherence tomography
}

Unterhuber, A.; Povaay, B.; Müller, André; Jensen, Ole Bjarlin; Duelk, M.; Le, T.; Petersen, Paul Michael; Velez, C.; Esmaeelpour, M.; Andersen, Peter E.

Total number of authors:

11

Published in:

Optics Letters

Link to article, DOI:

10.1364/OL.38.004312

Publication date:

2013

Document Version

Publisher's PDF, also known as Version of record

Link back to DTU Orbit

Citation $(A P A)$ :

Unterhuber, A., Povaay, B., Müller, A., Jensen, O. B., Duelk, M., Le, T., Petersen, P. M., Velez, C.,

Esmaeelpour, M., Andersen, P. E., \& Drexler, W. (2013). Simultaneous dual wavelength eye-tracked ultrahigh resolution retinal and choroidal optical coherence tomography. Optics Letters, 38(21), 4312-4315. https://doi.org/10.1364/OL.38.004312

\section{General rights}

Copyright and moral rights for the publications made accessible in the public portal are retained by the authors and/or other copyright owners and it is a condition of accessing publications that users recognise and abide by the legal requirements associated with these rights.

- Users may download and print one copy of any publication from the public portal for the purpose of private study or research.

- You may not further distribute the material or use it for any profit-making activity or commercial gain

- You may freely distribute the URL identifying the publication in the public portal 


\title{
Simultaneous dual wavelength eye-tracked ultrahigh resolution retinal and choroidal optical coherence tomography
}

\author{
A. Unterhuber, ${ }^{1, *}$ B. Považay ${ }^{1,2}$ A. Müller, ${ }^{3}$ O. B. Jensen, ${ }^{3}$ M. Duelk, ${ }^{4}$ T. Le ${ }^{5}$ P. M. Petersen, ${ }^{3}$ \\ C. Velez, ${ }^{4}$ M. Esmaeelpour, ${ }^{1}$ P. E. Andersen, ${ }^{3}$ and W. Drexler ${ }^{1}$ \\ ${ }^{1}$ Medical University of Vienna, Center for Medical Physics and Biomedical Engineering, Waehringer Guertel 18-20, \\ AKH-Wien E4.L, 1090 Vienna, Austria \\ ${ }^{2}$ Bern University of Applied Sciences, Institute for Human Centered Engineering, optoLab, Quellgasse 21, \\ CH-2502 Biel/Bienne, Switzerland \\ ${ }^{3}$ DTU Fotonik, Department of Photonics Engineering, Frederiksborgej 399, 4000 Roskilde, Denmark \\ ${ }^{4}$ EXALOS AG Wagistrasse 21, CH-8952 Schlieren, Switzerland \\ ${ }^{5}$ Femtolasers Produktions GmbH, Fernkorngasse 10, 1100 Vienna, Austria \\ ${ }^{*}$ Corresponding author: angelika.unterhuber@meduniwien.ac.at
}

Received July 18, 2013; accepted September 6, 2013;

posted September 23, 2013 (Doc. ID 194206); published October 18, 2013

\begin{abstract}
We demonstrate an optical coherence tomography device that simultaneously combines different novel ultrabroad bandwidth light sources centered in the 800 and $1060 \mathrm{~nm}$ regions, operating at $66 \mathrm{kHz}$ depth scan rate, and a confocal laser scanning ophthalmoscope-based eye tracker to permit motion-artifact-free, ultrahigh resolution and high contrast retinal and choroidal imaging. The two wavelengths of the device provide the complementary information needed for diagnosis of subtle retinal changes, while also increasing visibility of deeper-lying layers to image pathologies that include opaque media in the anterior eye segment or eyes with increased choroidal thickness. (C) 2013 Optical Society of America

OCIS codes: (170.4500) Optical coherence tomography; (170.4470) Ophthalmology; (140.3590) Lasers, titanium. http://dx.doi.org/10.1364/OL.38.004312
\end{abstract}

Optical coherence tomography (OCT) is an interferometric, well-established imaging modality obtaining depthresolved volumetric information [1]. In recent years, multiple developments such as low-cost, compact, ultrabroad light sources at different wavelengths combined with high-speed detector arrays $[\underline{1,2}]$ and tunable light sources together with low noise, high-speed detectors [3] and active tracking [4] or passive registering systems $[\overline{5}, 6]$ have been demonstrated to significantly improve OCT applications. Several of these key technologies have to be combined to meet the demands of modern clinical ophthalmic diagnosis of the posterior segment even in the presence of opaque ocular media (e.g., corneal haze, cataract), increased retinal or choroidal thickness, absorbing fundus pigmentation, and motion due to limited patient compliance or lack of fixation [7].

The two near-infrared (NIR) wavelength regions applicable for ophthalmic imaging of the posterior eye segment are 800 and $1060 \mathrm{~nm}$. Common clinical OCT devices perform raster scanning across the retina and stay well below the safety limits for a static beam [8]. Scanner motion and intrinsic eye motion of the subject, such as continuous drifts, rapid microsaccades, or saccades continuously reposition the beam relative to the retina and can strongly distort scan patterns with more than 10 $20 \mathrm{~ms}$ acquisition time. Common OCT systems have acquisition rates up to $\sim 100 \mathrm{kHz}$. Significantly higher acquisition rates have been reported where normal motion of the object is negligible. However, widespread clinical application is limited-especially for subjects with less transparent eye media, imperfect optics, and inability to fixate-due to their technical complexity. Therefore, it is still challenging to repeatedly monitor exactly the same position of tissue by unguided scanning at high image quality as demanded for precise diagnostics and long-term investigation in clinics.

In this Letter, we present a dual wavelength ophthalmic OCT device that features eye tracking and image registration, selection, and averaging that operates simultaneously at two wavelengths. About $170 \mathrm{~nm}$ in the $800 \mathrm{~nm}$ and $>110 \mathrm{~nm}$ in the $1060 \mathrm{~nm}$ wavelength region at FWHM (full width at half-maximum) were delivered by two new types of compact, cost-effective Ti:Sapphire lasers $[\underline{9}, 10]$ and a novel multiplexed, fiber-coupled superluminescent diode source (SLD). This system enables ultrahigh resolution (UHR) and enhanced contrast retinal and choroidal OCT $[11,12]$, together with a large penetration depth into the choroid.

The OCT engine was built upon the platform of a commercial Heidelberg Engineering (Heidelberg, Germany) Spectralis OCT device with patient interface, including a LED fixation target and tracking device. The built-in confocal scanning laser ophthalmoscope (SLO) operating at $735 \mathrm{~nm}$ corrects for eye motion in real time [4]]. For our experiments, the system was modified to support the bandwidth. Integration of the 800 and $1060 \mathrm{~nm}$ broadband light sources and the electronic coupling of the two associated frequency domain OCT engines enabled acquisition of cross-sectional scans at depth sampling line rates of $66 \mathrm{kHz}$ (see Fig. 1).

The standard Spectralis input module and the internal optics were replaced with specially designed broadband optics and beam splitters to support a spectral region between $650-1150 \mathrm{~nm}$. In standard configuration the system, which is equipped with a $47 \mathrm{~nm}$ broad SLD operating at $880 \mathrm{~nm}$, has $8 \mu \mathrm{m}$ axial resolution in air. In a 


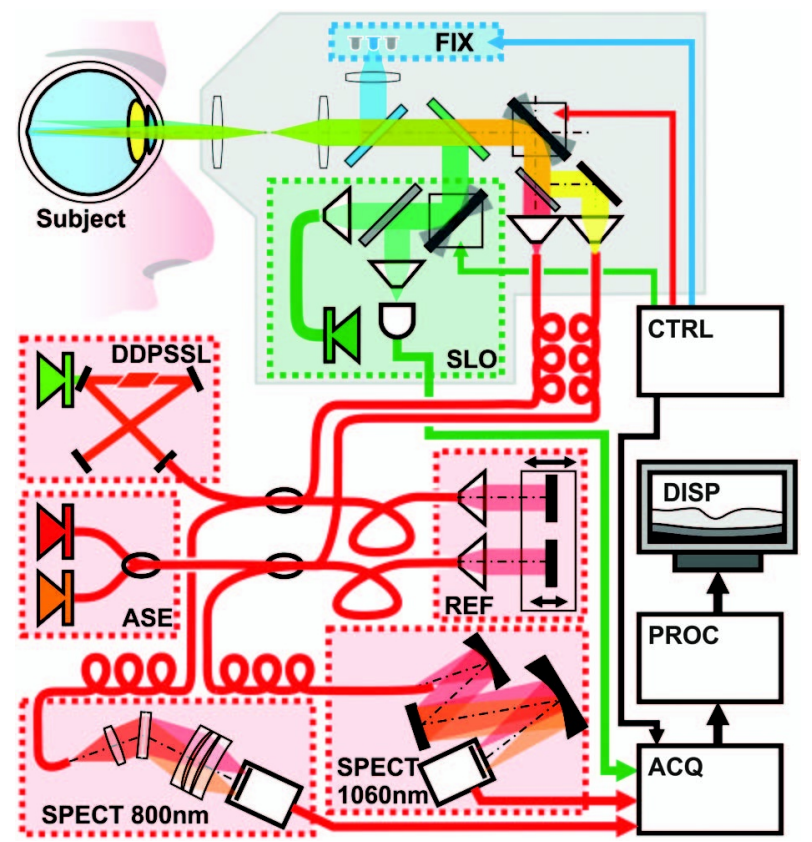

Fig. 1. Simultaneous dual wavelength, eye-tracked OCT. Fixation light (FIX) and scanning laser ophthalmoscope (SLO) control and correct eye motions via control hardware (CTRL). The original $880 \mathrm{~nm}$ SLD is paired with an amplified spontaneous emission source (ASE) at $1060 \mathrm{~nm}$. The detection via two spectrometers (SPECT) and a Camera Link acquisition system (ACQ) is processed (PROC) in real time and visualized on a display (DISP).

first step, a second channel with an amplified spontaneous emission source (ASE) operating in the $1060 \mathrm{~nm}$ wavelength region with approximately the same resolution was integrated to verify the performance in respect to the standard Spectralis. Keeping the commercial SLO tracker, both wavelength channels were externally integrated and upgraded to support wider bandwidth and higher resolution.

A schematic of the OCT engine is shown in Fig. 1. Two channels share a common patient interface with two specially designed dichroic beam splitters separating the fixation and SLO light from the two individually focusable collinear OCT beams (displaced by $\sim 100 \mu \mathrm{m}$ ) to limit the instantaneous power density onto the retina. The original free space reference arm of the Spectralis for adjusting the OCT imaging depth is separated into two external delay lines with fiber-coupled collimator and 800 and $1060 \mathrm{~nm}$ fiber-optic ports with two sets of polarization control paddles, attenuator, and reflector. The setup is kept flexible to allow for simultaneous and consecutive integration and testing of the novel light sources to investigate their applicability for UHR retinal and choroidal imaging. For broadband operation at $800 \mathrm{~nm}$, a CCD camera-based (Atmel Aviiva, E2V) transmission grating spectrometer was designed to support a bandwidth of $>250 \mathrm{~nm}$ while, for broadband operation at $1060 \mathrm{~nm}$, an all-reflective InGaAs camera-based Czerny-Turner-type spectrometer [13] was designed to facilitate a bandwidth of $>130 \mathrm{~nm}$. Due to the camera's (SUI-LDH2, Sensors Unlimited Inc., NJ, USA) pixel aspect ratio of $25 \times 500 \mu \mathrm{m}$, the elongated point spread functions resulting from the astigmatism introduced by the two off-axis positioned spherical mirrors are effectively captured without losses.

The spectrometers are aligned toward optimum spectral resolution and throughput by an external dispersionless, free-space interferometer set to a large delay between sample and reference arm. The high spectral beating frequency corresponds to a spatial offset between the two arms at $60 \%-80 \%$ of the imaging range. The same interferometer is utilized for calibration of the nonlinear resampling function that converts the spectrometer pixel coordinates to the appropriate vectors in $\mathrm{k}$-space [14]. The depth-dependent roll-off is $-13.8 \mathrm{~dB}$ at the $2.66 \mathrm{~mm}$ long sampling depth range measured in air. The internal Spectralis light source is replaced by a compact Ti:Sapphire pumped by a simple and robust frequency-doubled, directly diode pumped solid state laser (DDPSSL) with $90 \mathrm{~nm}$ bandwidth at FWHM and a new type of Ti:Sapphire laser (INTEGRAL Core, Femtolasers) that delivers sub-10-fs pulses at significantly reduced weight and cost over state-of-the-art fs lasers, with comparable pulse durations with more than $170 \mathrm{~nm}$ bandwidth at FWHM to increase the axial resolution (to $\sim 3$ and $\sim 1.7 \mu \mathrm{m}$, respectively, in air). The uniqueness of the former Ti:Sapphire is the pump of a diode-based system with good beam quality, intensity stability, and low noise $[9,10]$. Using dispersive mirrors, a very efficient and stable laser that accepts cost-effective small pump lasers and operates at high repetition rates $(>300 \mathrm{MHz})$ could be released and disproves the tendency that high repetition rate broadband lasers usually need larger pump lasers. The additional longer wavelength channel is equipped with a $52 \mathrm{~nm}$ ASE source operating in the $1060 \mathrm{~nm}$ region (BBS-1 $\mu \mathrm{m}$, Multiwave Photonics SA, Portugal). To increase resolution, a multisource SLD (EBS4C34, EXALOS, Switzerland) with $20 \mathrm{~mW}$ output power and $111 \mathrm{~nm}$ bandwidth (FWHM) was specially tailored for the $1060 \mathrm{~nm}$ water transparency window [see Fig. 2(e)].

During operation, the external cameras are triggered by the internal SLO signals that also control the galvanometric mirror scanners. The spectral line exposure time operates at $66 \mathrm{kHz}$ camera-line rate, thereby approximately doubling the depth-scan rate of the commercial device. Images are acquired with the system-specific 1538 depth scans per frame in stacks of up to 200 frames. The key to high contrast at high resolution lies in the registration and selection of corresponding depth scans. Usually, 20-100 frames can be used to improve sensitivity without losing details. This value is strongly dependent on the amount of motion and resolution. Further averaging can improve the sensitivity, but this is dependent on the availability of well-matched frames or comes at the price of reduced resolution. Especially for UHR OCT, there is a critical trade-off between number of frames and resolution. Subjects are measured in compliance with the declaration of Helsinki, with the admission of the local ethics committee according to European medical product regulations. The maximum power measured at the cornea is held below $0.8 \mathrm{~mW}$ at $800 \mathrm{~nm}$, below $1.2 \mathrm{~mW}$ at $880 \mathrm{~nm}$, and below $3 \mathrm{~mW}$ in the $1060 \mathrm{~nm}$ wavelength region, well below the safety limits [15]. The 800 and $1060 \mathrm{~nm}$ beams are laterally displaced by $0.2 \mathrm{~mm}$ for safety reasons. The system is secured by a shutter 
mechanism that interrupts the beam in case of scanner failure by shutting down if the scan angle sinks below $13.5^{\circ}$ of visual angle $(\sim 3.6 \mathrm{~mm}$ on the retina) within a frame $(<30 \mathrm{~ms})$. The SLO and the tracking mechanism help to locate the correct retinal alignment and automatic repositioning, and keep the overall alignment time typically well under 5 min even for untrained or less compliant subjects. Axial motions are sufficiently suppressed by a forehead and chinrest to keep the fundus within a third of the detection range. The sensitivity of the system was evaluated with an Ag mirror measured close to the zero delay and showed comparable results for $800 \mathrm{~nm}(83 \mathrm{~dB})$ and around $1060 \mathrm{~nm}(84 \mathrm{~dB})$. Averaging of 100 frames yielded an overall sensitivity of $103 \mathrm{~dB}$. The large deviation from the theoretically achievable values without eye and optics of $118 \mathrm{~dB}$ for a single frame and 138 for 100 tomograms is due to significant losses caused by the attenuation of the OCT signal due to water absorption, broadband optics, aberrations, losses and polarization mismatch, and by the fact that the two spectrometer designs are not optimized for one specific light source but for different light sources varying in center wavelength and bandwidth.

During examination of a human subject, the beam is centered onto the pupil, followed by focusing the SLO and fine lateral positioning of the SLO beam by optimizing the illumination for peak intensity and flatness of illumination. The reference arm length then has to be adjusted to place the point of highest sensitivity at the zero-delay position on the distal side of the depth scan, which is the sclera in this case. This inverted object placement partially compensates the depth-dependent signal roll-off of the spectrometer with the stronger back reflections obtained from the surface layers of the sample. Figures 2(a)-2(d) display tomograms obtained at approximately the same location with different light sources. Here, (a) UHR and (b) standard resolution OCT at $800 / 880 \mathrm{~nm}$ are compared with (c) simultaneous acquired standard and (d) high-resolution OCT at $1060 \mathrm{~nm}$ and clearly visualize the difference in penetration, contrast, and resolution according to the light source parameters. For registration the Lucas-Kanade method is applied together with a statistical analysis based on a similarity measure for elimination of unsuitable frames. The individual frames are superimposed, allowing for linear shifts and distortions between each other and averaged with a median filter.

The tomograms in Fig. 3 reveal the difference in resolution between (a) standard Spectralis and, (b) and (c), Ti:Sapphire-based OCT systems. Extending the system performance from standard resolution OCT enables despeckling at ultrahigh axial resolution and consequently helps to delineate structures not visible in a tracked standard resolution system, such as better contrasting of the ganglion cell layer to the inner plexiform layer and a finer detailing of photoreceptor morphology and the separation of the RPE-choriocapillaris complex fine structure. Figures 3(b) and 3(c) show the trade-off between moderate $(>90 \mathrm{~nm}$, DDPSSL) to ultrabroad bandwidth $(>170 \mathrm{~nm}$, INTEGRAL Core) and increase in resolution and enhanced visualization of details.

Figures $\underline{4(a)}$ and $\underline{4(b)}$ display tomograms of the same individual obtained at the same location with the ASE
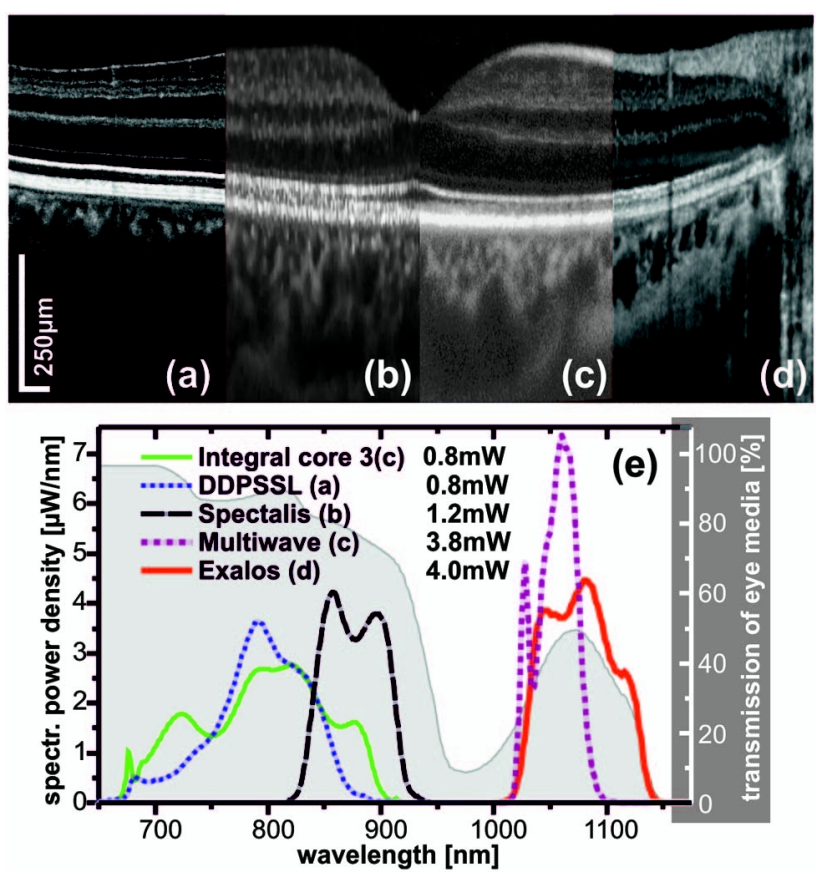

Fig. 2. Comparison of (a) multiple sampled and tracked UHR, (b) standard resolution OCT at 800/880 nm with (c) simultaneously acquired standard, and (d) high resolution $1060 \mathrm{~nm}$ OCT. (e) Power spectral density of applied lasers, corrected for double passing the eye (gray): INTEGRAL core (green, solid), DDPSSL (blue, dotted), SLD (black, dashed), ASE source (magenta, dotted), and multiplexed SLD (red, solid) for imaging in water transparency window around $1060 \mathrm{~nm}$.
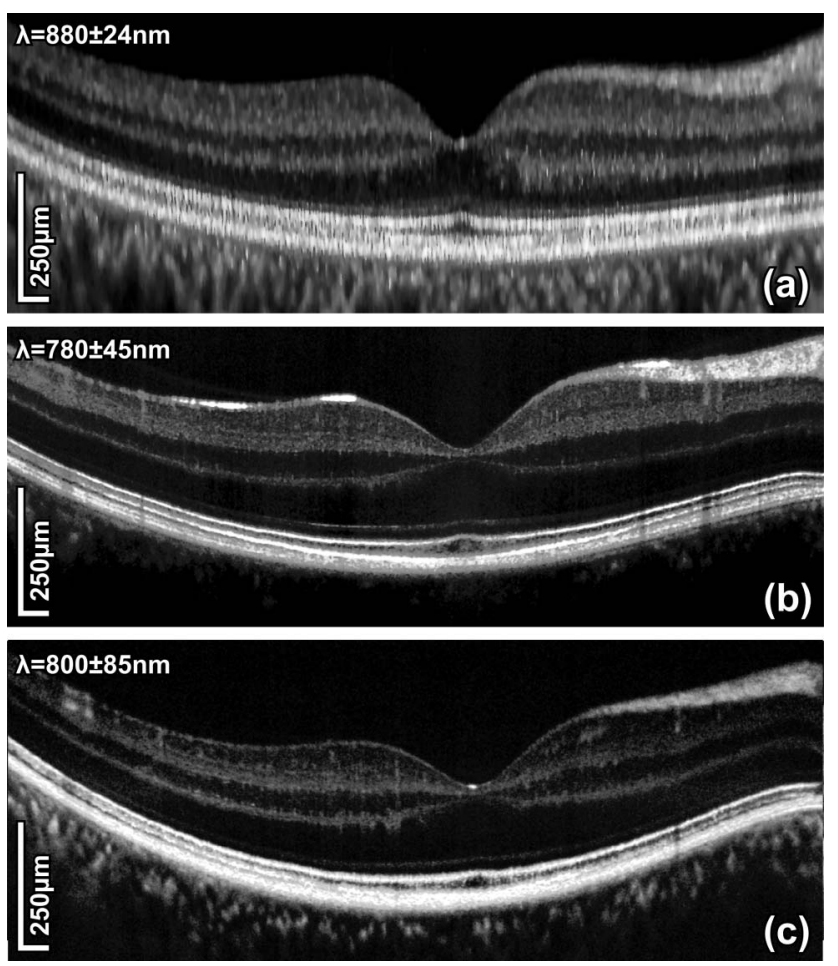

Fig. 3. Tomograms utilizing the retracking function of the device with (a) internal light source (100 frames) compared with (b) DDSSL (50 frames) and (c) INTEGRAL core (20 frames). Tomograms are displayed in a 1:3 ratio (horizontal:vertical) for better visualization of the layered structure. 

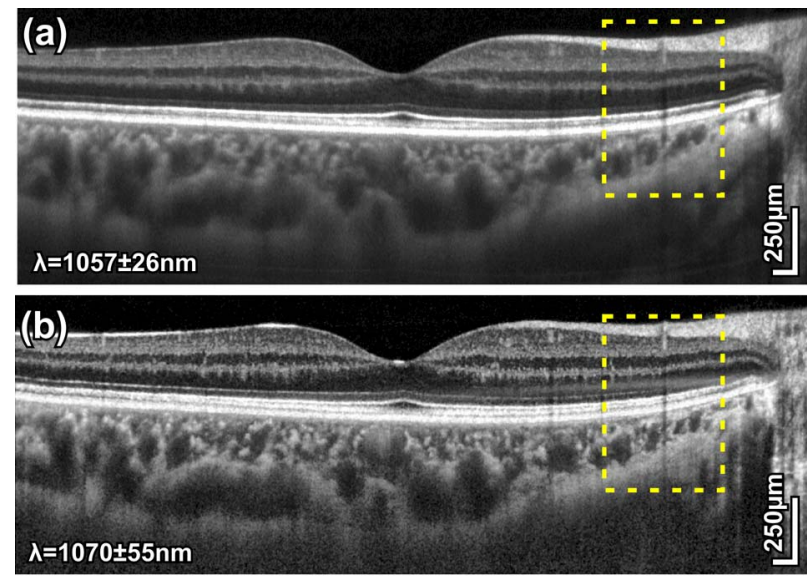

(c)
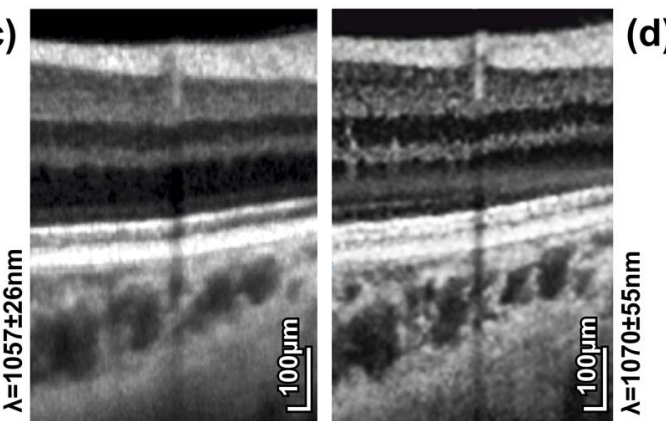

Fig. 4. Tomograms (1:3 ratio) of the same subject acquired at the same location utilizing the retracking function. Averaged tomograms acquired with (a) ASE and (b) SLD. (c) and (d) magnification of the demarcated regions in (a) and (b) unveil the enhanced level of detail obtained by improved registration and increased resolution in the retina and choroid, despite the lower number of averaged frames and the increased granularity caused by remaining speckle.

compared to the multiplexed SLD in the $1060 \mathrm{~nm}$ wavelength region with increased penetration into the choroid compared to the $800 \mathrm{~nm}$ images. The increased bandwidth results in an expected increase of resolution, which is shown in the zoom in Figs. 4(c) and 4(d). Nonetheless, axial resolution is reduced by nonlinear sampling of the spectrum, modulations in the effective spectrum, and chromatic dispersion as well as non-Gaussian spectra. Hence, sampling and k-mapping are highly optimized, and the spectral shape is apodized to a Gaussian fit to the average spectrum while second- and third-order dispersion is dynamically compensated for in each image set, leading to speckle-limited resolution in the single frame [14] and unveiling the enhanced level of detail obtained by improved registration and increased resolution.

In conclusion, we demonstrated a dual wavelength UHR, SLO-based, eye-tracked OCT system simultaneously operating in the 800 and $1060 \mathrm{~nm}$ wavelength region with novel ultrabroad bandwidth and cost-effective light sources. The fiber-based sources deliver superior spectral bandwidth in a compact format that can match the results obtained with significantly more costly large-scale lasers. By obtaining high-quality tomograms, with a penetration depth of up to $500 \mu \mathrm{m}$ into the choroid, that could be arbitrarily selected and automatically repositioned to the location of interest during multiple measurements or in follow-up studies, this instrument could be perfectly suited for clinical investigation.

This research was supported by a Marie Curie Intra European Fellowship within the 7th European Community Framework Programme, Medical University Vienna, European project FAMOS (FP7 ICT 317744), Bern University of Applied Sciences, Swiss National Science Foundation, OENB Jubilaeums fond 14294, FWF-NFN "Photoacoustic imaging in Biology and Medicine," and the Christian Doppler Society (Christian Doppler Laboratory "Laser development and their application in medicine"). The authors also gratefully acknowledge Heidelberg Engineering for equipment support.

\section{References}

1. W. Drexler and J. G. Fujimoto, Optical Coherence Tomography (Springer, 2008).

2. W. Drexler, U. Morgner, R. K. Ghanta, F. X. Kärtner, J. S. Schuman, and J. G. Fujimoto, Nat. Med. 7, 502 (2001).

3. T. Klein, W. Wieser, C. M. Eigenwillig, B. R. Biedermann, and R. Huber, Opt. Express 19, 3044 (2011).

4. D. X. Hammer, R. D. Ferguson, J. C. Magill, M. A. White, A. E. Elsner, and R. H. Webb, Appl. Opt. 42, 4621 (2003).

5. A. R. Fuller, R. J. Zawadzki, S. Choi, D. F. Wiley, J. S. Werner, and B. Hamann, IEEE Trans. Vis. Comput. Graphics 13, 1719 (2007).

6. B. Antony, M. D. Abràmoff, L. Tang, W. D. Ramdas, J. R. Vingerling, N. M. Jansonius, K. Lee, Y. H. Kwon, M. Sonka, and M. K. Garvin, Biomed. Opt. Express 2, 2403 (2011).

7. J. S. Schuman, C. A. Puliafito, and J. G. Fujimoto, Optical Coherence Tomography of Ocular Diseases, 2nd ed. (Slack, 2004).

8. Y. Huang, S. Gangaputra, K. E. Lee, A. R. Narkar, R. Klein, B. E. K. Klein, S. M. Meuer, and R. P. Danis, Investig. Ophthalmol. Vis. Sci. 53, 2133 (2012).

9. A. Müller, O. B. Jensen, A. Unterhuber, T. Le, A. Stingl, K.-H. Hasler, B. Sumpf, G. Erbert, P. E. Andersen, and P. M. Petersen, Opt. Express 19, 12156 (2011).

10. O. B. Jensen, P. E. Andersen, B. Sumpf, K.-H. Hasler, G. Erbert, and P. M. Petersen, Opt. Express 17, 6532 (2009).

11. A. Unterhuber, B. Považay, B. Hermann, H. Sattmann, A. Chavez-Pirson, and W. Drexler, Opt. Express 13, 3252 (2005).

12. M. Esmaeelpour, B. Považay, B. Hermann, B. Hofer, V. Kajic, K. Kapoor, N. J. L. Sheen, R. V. North, and W. Drexler, Investig. Ophthalmol. Vis. Sci. 51, 5260 (2010).

13. B. Považay, B. Hermann, B. Hofer, V. Kajic, E. Simpson, T. Bridgford, and W. Drexler, Investig. Ophthalmol. Vis. Sci. 50, 1856 (2009).

14. B. Hofer, B. Považay, B. Hermann, A. Unterhuber, G. Matz, and W. Drexler, Opt. Express 17, 7 (2009).

15. ICNIRP, "Revision of the Guidelines on Limits of Exposure to Laser Radiation of Wavelengths between $400 \mathrm{~nm}$ and $1.4 \mu \mathrm{m}, " 2000$. 